\title{
The ASTERICS project: developing a new era of Multi-messenger astrophysics
}

\section{Carole A. Jackson ${ }^{1}$}

ASTRON, The Netherlands Institute for Radioastronomy

Oude Hoogeveensedijk 4, Dwingeloo, The Netherlands

E-mail: jacksoneastron.nI

\section{Giuseppe Cimò}

Joint Institute for VLBI ERIC

Oude Hoogeveensedijk 4, Dwingeloo, The Netherlands

E-mail: cimoljive.eu

\section{Rob L.J. van der Meer}

ASTRON, The Netherlands Institute for Radioastronomy Oude Hoogeveensedijk 4, Dwingeloo, The Netherlands

E-mail: meereastron.nI

EU H2020 project “ASTERICS" (2014-2019) establishes a collaborative cluster to develop systems, tools and techniques for four next generation ESFRI telescope facilities in astronomy, astrophysics and astroparticle physics. In focusing on ELT, SKA, KM3NeT and CTA, ASTERICS achieved its aims. As outlined in this paper and described in detail in the papers of this publication, it solved key challenges common to the ESFRIs and adopted cross-cutting solutions with mutual and wide-ranging benefit to all concerned. ASTERICS facilitated the process of identifying areas ready for rapid technology development, where the adoption of a complementary and synergetic approach across the ESFRI projects could lead to significant added value in their operational phase.

The New Era of Multi-Messenger Astrophysics - Asterics2019

25 - 29 March, 2019

Groningen, The Netherlands 


\section{ASTERICS - aiming to maximise the science from the 2020 ESFRIs}

The ASTERICS project (Astronomy ESFRI and Research Infrastructure Cluster) is a collaborative cluster for the next generation ESFRI telescope facilities and other relevant research infrastructure initiatives in the area of astronomy, astrophysics and astroparticle physics.

The ASTERICS project was timely given the significant European involvement in the construction of new large global facilities illustrated in Figure 1, namely

- $\quad$ Square Kilometre Array (SKA), observing at radio wavelengths,

- Cherenkov Telescope Array (CTA), observing extremely high energy gamma rays,

- Cubic KiloMetre (km3) Neutrino Telescope (KM3NeT), observing neutrinos from the universe

- $\quad$ Extremely Large Telescope (ELT), observing at optical wavelengths.
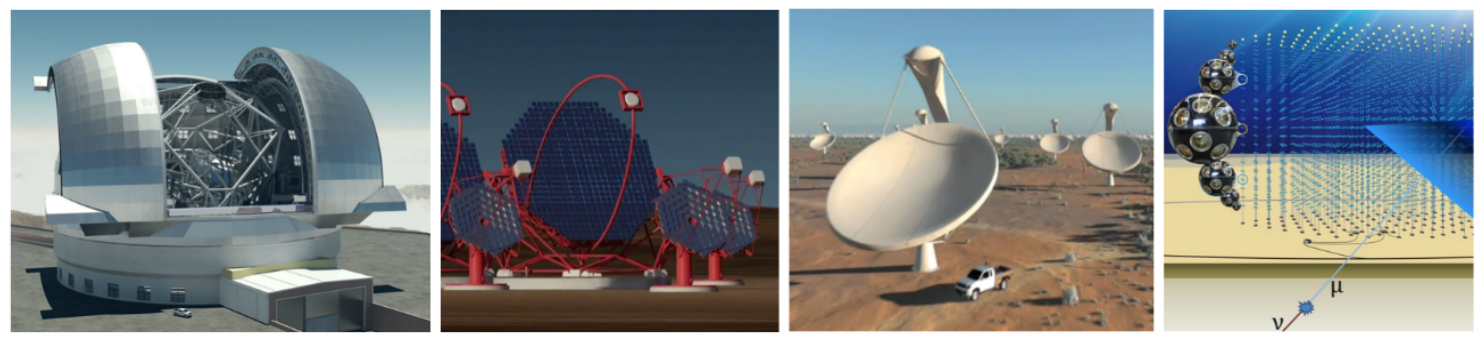

Figure 1: ESFRI facilities (ELT, CTA, SKA, KM3NeT)

Each of these is a mega-scale Research Infrastructure, endorsed and prioritised by the European Strategy Forum on Research Infrastructures (ESFRI). During the later stages of ASTERICS, the European Solar Telescope was included as an additional ESFRI facility. These ESFRI projects, along with their pathfinders, exploit our observational capabilities in new ways: they allow us to probe the Universe in combination with the detection of neutrinos and high-energy particle showers. By combining their capabilities, we are working towards true multi-messenger astronomy and opening unexplored regions of a combined observational parameter space.

Ensuring that Europe, and the world, fully exploits the impact of the ESFRI facilities, demands close integration through the science lifecycle of each (namely operations, (data) processing, and analysis and data inter-operability).

Before this project created the ASTERICS collaborative cluster, interactions between the communities involved in the development of these ESFRI facilities were limited. Given each of these new facilities will generate vast amounts of data, ASTERICS focused on key aspects of

- data handling - the generation, transport, preservation, retrieval and analysis,

- interoperability between facilities - linked to smart analysis, scheduling for simultaneous observations, and

- fast response of each facility.

Education, training and interacting with ESFRI facility staff, industry and the wider user community were core to ASTERICS' activities in a spirit of "open innovation". These activities defined requirements, and ensured wide and rapid up-take of ASTERICS' results and deliverables. A final, but important ASTERICS goal was to engage with society and the general public via new and exciting citizen science applications.

\section{The ASTERICS project - core challenges and outputs}

The ASTERICS Consortium comprised 26 partners, universities and research institutes, each linked to one or more of these ESFRI facilities. The first step for ASTERICS was to bring together the 
communities: These had worked almost independently until ASTERICS' existence. Fundamental to ASTERICS was bringing together individuals and groups in specially organised meetings, workshops and events at all levels. These events included the use of targeted invitations and travel support to ensure key participation. ASTERICS collaboration helped define thematic meetings targeted to specific tasks. In this way, ongoing collaborations, visits and co-organising further events were seeded. The wide variety of development tasks within ASTERICS inevitably led to a large number of events: these were spread over each calendar year to enable maximum participation.

Many tasks within ASTERICS engaged with commercial partners (industry). As a result, communication and citizen science were core to our activities. Therefore, as well as the many specialist events noted above, we also set out to widely communicate the results of ASTERICS: this included general PR, popular and peer reviewed articles and participation in many international seminars and conferences.

At the request of ASTRONet, the ASTERICS Policy Forum conducted a study of the science cases for the ASTERICS ESFRI facilities. This was to identify the challenges to delivering multi-messenger astrophysics across the facilities in the 2020's. The Policy Forum determined that there are some significant strategic questions to ensure the success of the ESFRIs in this sphere, for example, who pays for the common challenges and who coordinates the matching of facility and user requirements? The outcome of the work of the Policy Forum is recorded in Mourard et al [1].

In the remainder of this paper we summarise the four core work packages of ASTERICS. More details to the results are given in the papers of these proceedings.

\section{DECS: Dissemination, Engagement and Citizen Science}

ASTERICS work-package DECS was dedicated to disseminating our results to as wide an audience as possible. This was achieved via the production of high-quality outreach materials and direct engagement with our stakeholders. Citizen Scientist Mass Participation Experiments were used to capture the interest of the general public.

Four citizen science projects were developed during ASTERICS, namely

- Muon Hunter (I and II) [2]

- CREDO [3,4]

- Euclid: Challenge the Machines [5]

- $\quad$ SuperWASP Variable Stars [6] (home web page shown in Figure 2)

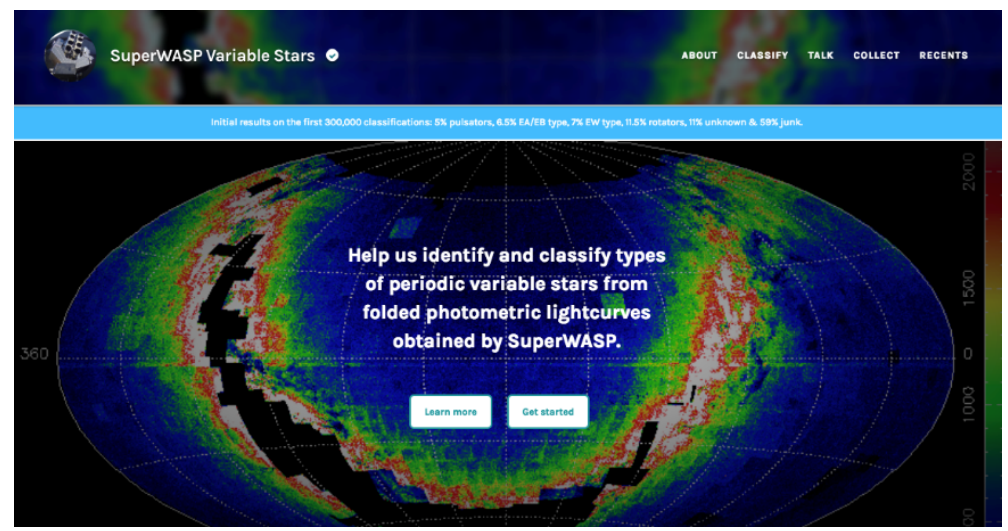

Figure 2: Zooniverse page of the citizen science experiment SuperWASP

A number of videos were created to engage the general public in the project activities. In the series "60 second adventures in collaborative science", David Mitchell, a well-known UK actor, narrates the concepts of Citizen Science, Messengers from Space and Multi-Messenger Science [7]. 


\section{OBELICS: OBservatory E-environments Linked by common ChallengeS}

ASTERICS work-package OBELICS focussed on enabling interoperability and software re-use in data generation, integration, and analysis. These factors are key to ASTERICS: each of the ESFRIs faces "big data challenges" in the data flow from observations to scientific data products.

The first step for OBELICS was to create a community for multi-messenger data analysis. Three workshops led to new academic and industrial collaborations. In addition, three summer schools trained more than 200 students and researchers to the use of modern data analysis tools, from the basics of Python to parallel computing to GPU programming (Figure 3).

This work package has also been successful in producing common solutions for the ESFRIs. Software libraries were developed through creating bridges and cooperative links between the scientific communities, European e-infrastructures initiatives, other consortia and institutions provided state of the art expertise in data management and computing through a number of general workshops.

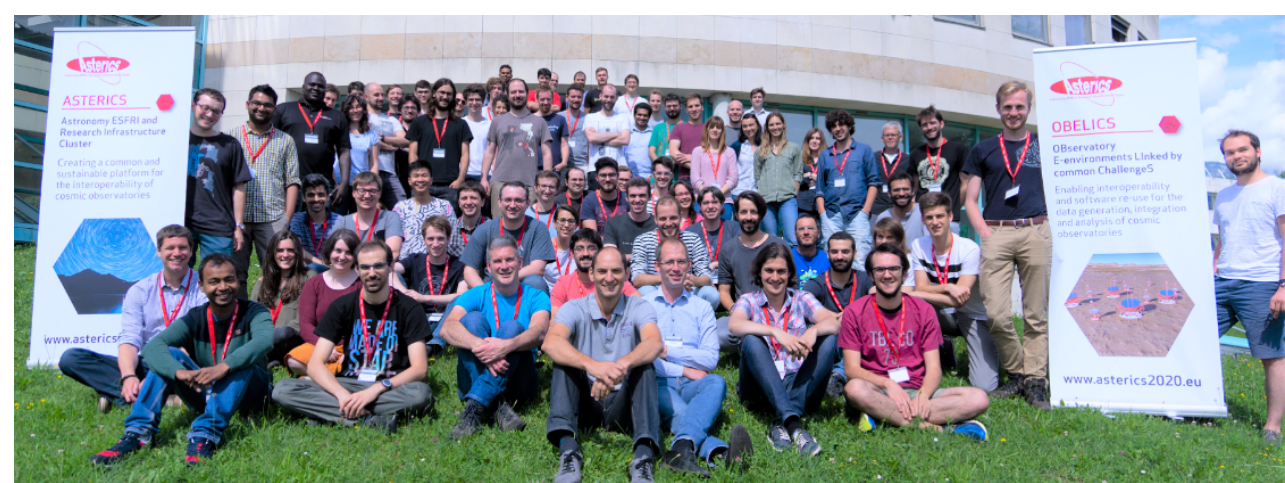

Figure 3: Participants of the second programming school in Annecy (France, 4-8 June 2018)

The OBELICS partners have produced a repository of statistically robust and domain independent open source software libraries for data analysis and data mining on Peta-scale datasets. The repository also includes a series of services providing a framework for the analysis and integration of data for large-scale infrastructures [8]. OBELICS also initiated a number of industrial collaborations. These collaborations delivered a number of outcomes, including a prototype ASTRON data portal, a modular data reduction framework for astronomy pipelines, a method to automatically detect Solar Radio Bursts using a novel Machine Learning technique, "Wavefier" - a prototype for a real time Gravitational Wave transient signal classifier and GammaLearner which developed deep learning methods for CTA.

\section{DADI: Data Access, Discovery and Interoperability}

ASTERICS work-package DADI addressed the challenges regarding the access to ESFRI data. The goal of the DADI work-package was to make the ESFRI and pathfinder project data available for discovery and use by a wide astronomical community, interoperable in a homogeneous international framework, and accessible with a set of common tools. The main tool for such interoperability is the Virtual Observatory (VO) framework. ASTERICS has successfully fostered the participation of ESFRI projects in the development of the underlying interoperability framework and has been essential to continue develop a framework based on the international Virtual Observatory standards..

The work of the DADI work-package advanced the preparation for interoperable ESFRI data discovery and access. The ASTERICS all-sky multi-dimensional approach has led to spectacular new data visualisation capabilities, which have been implemented in observatory archive services and for gravitational wave tools (GWsky [9]). New standards to describe data provenance, essential for data reusability and trustworthiness, were created [10]. ASTERICS-DADI has worked in adapting the VO to 
the needs of the ESFRI facilities, also supporting and training them in use and implementation of the VO. In parallel, the training and support of the astronomical community in the use of the VO was by through a number of dedicated schools and workshops [11]. These workshops included the development and interaction with virtual reality applications that use the VO as illustrated in Figure 4).

As a result, the ESFRI telescopes and pathfinders emerge as consumers and actors of the VO. The ESFRI facilities have impacted the VO standards by being part of the development of tools and participating in discussions on requirements, reference implementations and prototype interfaces. Specifically, the developments have impacted Multi-Dimension data standards, All-sky approach, Time Domain astronomy, and Provenance.

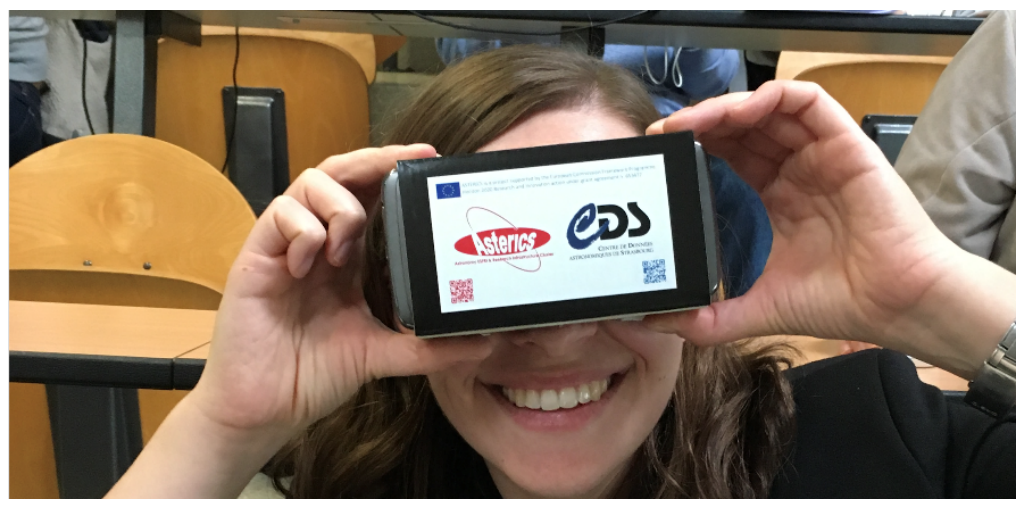

Figure 4: The virtual Observatory meets virtual reality

\section{CLEOPATRA: Connecting Locations of ESFRI Observatory and Partners in Astronomy for Timing and Real-time Alerts}

ASTERICS work-package CLEOPATRA set out to solve the critical (data) communication challenge faced by massive arrays of interconnected elements. Future instruments like the CTA and SKA will contain even larger numbers of elements. The number of antennas and their geographical distribution create new challenges with regard to time synchronisation, triggering fast response, and accessing large data streams. The activities of CLEOPATRA addressed these issues via the development of synergetic observing modes, and fast and reliable access to large data streams.

A workshop on alerting mechanisms from radio to $\gamma$-ray was organized in Amsterdam in September 2017 to define the current landscape of multi-facility collaborations. During this meeting, ESFRI representatives and the wider scientific community discussed how to optimize alert systems and followups in the era of multi-messenger astrophysics. Fast response is fundamental for triggered observations and the work-package contributed to the development of rapid response capability for LOFAR and the EVN. Trigger observations require real-time changes to the observing schedule of a telescope, which can be a hard problem to solve in case of a dynamically scheduled array of hundreds of antennas (e.g. CTA, SKA). As a result, ASTERICS developed a new and advanced scheduling algorithm for complex arrays.

ASTERICS-CLEOPATRA also contributed to improving the stability and calibration of White Rabbit, a technology that allows time distribution across long distances [12]. The White Rabbit hardware has been tested in a harsh environment in the Tunka valley (Siberia). Further successful VLBI/White Rabbit experiments were completed in transferring a Hydrogen maser signal via public fibre [13].

\section{Acknowledgements}

The ASTERICS project acknowledges funding from the European Commission Framework Programme Horizon 2020 Research and Innovation action under grant agreement n. 653477. We thank the original 
and interim project coordinators $\mathrm{M}$ Garrett and $\mathrm{M}$ de Vos for their significant effort in steering the work during its earliest phases.

\section{References}

[1] Mourard et al, PoS(Asterics2019)055

[2] https://www.zooniverse.org/projects/dwright04/muon-hunters-2-dot-0

[3] https://credo.science/

[4] Homola et al, PoS(Asterics2019)034

[5] https://www.zooniverse.org/projects/hughdickinson/euclid-challenge-the-machines

[6] https://www.zooniverse.org/projects/ajnorton/superwasp-variable-stars

[7] https://www.youtube.com/playlist?list=PLhQpDGfX5e7A0De8Cz_huUGSxiaQ9sfr4

[8] http://repository.asterics2020.eu/software

[9] Greco et al, PoS(Asterics2019)031

[10] Genova et al, PoS(Asterics2019)028

[11] Solano et al, PoS(Asterics2019)075

[12] Serrano et at, The White Rabbit Project in Proceedings of ICALEPCS TUC004, Kobe, Japan, 2009

[13] Boven et al, PoS(Asterics2019)017 